\title{
Risk factors for bovine herpesvirus-1 seropositivity
}

\author{
F. Boelaert ${ }^{\mathrm{a}, *}$, N. Speybroeck ${ }^{\mathrm{b}}$, A. de Kruif ${ }^{\mathrm{c}}$, M. Aerts ${ }^{\mathrm{d}}$, \\ T. Burzykowski ${ }^{\mathrm{d}}$, G. Molenberghs ${ }^{\mathrm{d}}$, D.L. Berkvens ${ }^{\mathrm{b}}$ \\ ${ }^{a}$ Co-ordination Centre for Veterinary Diagnostics, Veterinary and Agrochemical Research Centre, \\ Groeselenberg 99, 1180 Brussels, Belgium \\ ${ }^{\mathrm{b}}$ Institute for Tropical Medicine, Nationalestraat 155, 2000 Antwerp, Belgium \\ ${ }^{\mathrm{c}}$ Department of Reproduction, Obstetrics and Herd Health, Faculty of Veterinary Medicine, \\ Salisburylaan 133, 9820 Merelbeke, Belgium \\ ${ }^{\mathrm{d}}$ Centre for Statistics, Limburgs Universitair Centrum, Universitaire Campus, 3590 Diepenbeek, Belgium
}

Received 25 March 2004; received in revised form 30 November 2004; accepted 16 February 2005

\begin{abstract}
This paper reports the investigation of risk factors for bovine herpesvirus-1-seropositivity, based on a cluster-sample survey of the Belgian cattle population. This serosurvey was carried out in 1998 in 309 randomly selected unvaccinated herds of all types (dairy, mixed and beef) were all bovids $(N=11,284)$ were sampled.

Older and male cattle had higher seroprevalence. Origin (homebred or purchased) and herd size interacted; for smaller herds ( $\leq 50$ cattle on the premises), purchase status and larger herd size were risk factors, whereas these effects were not observed for larger herds.
\end{abstract}

(C) 2005 Elsevier B.V. All rights reserved.

Keywords: Cattle-microbiological diseases; Bovine herpesvirus-1; Infectious bovine rhinotracheitis; Seroprevalence; Risk assessment; Belgium

\section{Introduction}

Bovine herpesvirus-1 (BHV-1) infection is a worldwide contagious disease of economic and trade importance. Hence, it is a disease on the B List of the World Organisation for

\footnotetext{
* Corresponding author. Present address: European Food Safety Authority (EFSA), Monitoring of Zoonoses, Largo N. Palli 5/A, I-43100 Parma, Italy. Tel.: +32 2379 0628/+39 0521 036 466; fax: +32 2379 0401/+390521036 566.

E-mail addresses: frboe@var.fgov.be, frank.boelaert@efsa.eu.int (F. Boelaert).
} 
Animal Health (O.I.E., 2004). Programs to eradicate BHV-1 have been implemented in several European countries to facilitate the free trade of cattle, semen and embryos within the European community. The BHV-1 seroprevalence in the Belgian cattle population was determined in 1998 by a serological survey (Boelaert et al., 2000). In 309 unvaccinated herds, the overall herd seroprevalence was $67 \%(95 \% \mathrm{CI}=62,72)$. A design-based analysis, taking into account the effects of clustering and stratification estimated the animal-level seroprevalence to be $36 \%(95 \% \mathrm{CI}=30,42)$ (Speybroeck et al., 2003). As those authors reported, clustering is a key feature of the BHV-1 1998-serosurvey data, with an intra-cluster (intra-herd) correlation coefficient of 0.64. Exposure to infectious agents probably results in a more-homogeneous serological status of animals within clusters as measured by the intra-cluster correlation coefficient (Donald and Donner, 1987; Rothman, 1990), because the interrelatedness of incidence and prevalence is the very characteristic that defines infectious diseases (Koopman et al., 1991; Susser, 1994a; De Jong, 1995). Consequently, it cannot be assumed that the animals are independent units (Schukken et al., 1991; Elbers and Schukken, 1995; Cameron and Baldock, 1998), because compared to other population members, animals within clusters have a higher chance of becoming infected once the infection is introduced into the herd.

In the literature, only herd-level BHV-1-seroprevalence (proportion of seropositive herds) risk factors of Dutch herds with dairy cows have been reported. van Wuijckhuise et al. (1998) found that larger herd size, dairy herds with beef/veal animals, and higher density of herds in the municipality were associated with higher herd-level BHV-1seroprevalence. Also, for farms with both dairy and beef/veal animals, there was a weak association between the purchase of stock and BHV-1 status. For pure dairy herds, the probability of having a negative or weakly positive BHV-1 status decreased linearly with the numbers of purchased stock. Also, in the Netherlands, van Schaik et al. (1998) reported that BHV-1-seropositive herds purchased cattle and participated in cattle shows more often, compared to BHV-1-negative herds. BHV-1-seropositive herds also had more (professional) visitors who used protective farm clothing less often. BHV-1-positive herds were situated closer to other cattle herds compared to BHV-1-negative herds. Failure of professional visitors (e.g. veterinarians, insemination technicians) to wear protective farm clothing when handling cattle and cattle escaping and mingling with other cattle were risk factors for introduction of BHV-1 onto BHV-1 free dairy herds (van Schaik et al., 2001).

Our objective was to investigate the 1998-survey results of the Belgian cattle population for animal- and herd-level risk factors associated with BHV-1-seropositivity.

\section{Materials and methods}

\subsection{The BHV-1-serosurvey data}

A detailed description of the BHV-1-serosurvey can be found elsewhere (Boelaert et al., 2000; Haine et al., 2004). Briefly, within each of the 10 provinces in Belgium, $1 \%$ of the total number of herds was selected randomly from SANITEL-cattle, the central computerized database for the identification and registration of the Belgian cattle population (Federal Public Service Health, Food Chain Safety and Environment, Belgium). 
The selection device was a software random-generator function of Visual Basic 3.0 (Microsoft Corp., 1993). Because all animals in the selected herds were blood sampled, this was a one-stage cluster-sample survey. The BHV-1-survey database comprised 11,284 cattle living in 309 unvaccinated herds.

Blood samples were tested for the presence of antibodies to glycoprotein B $(\mathrm{gB})$ (Kramps et al., 1994) of BHV-1, using a commercially available blocking ELISA (HerdChek $^{\circledR}$, Idexx, France). No published data of the diagnostic sensitivity and specificity of this serological assay exist. Kramps et al. (1994) estimated the sensitivity of an analogous non-commercial gB-blocking ELISA to be $99 \%$, whereas its diagnostic specificity in a BHV-1 free and unvaccinated animal population ranged from $96 \%$ (Kramps et al., 1994) to $99.7 \%$ (de Wit et al., 1998).

The BHV-1-survey database comprised the following covariates. The animal-level factors were age, sex and purchased (yes/no), which all were known from the SANITEL herd inventories. The herd-level covariates were herd type (dairy, mixed or beef) and herd size (number of cattle on the premises) and were recorded during the herd visit by the veterinary practitioners conducting the sampling. Beef type was defined as a cow-calf herd, a young-stock herd, a fattening-cow herd, a fattening-steer herd, a fattening-calf herd or a hobby holding. Mixed type represents herds with both dairy and beef cattle on the premises. The veterinary practitioners also interviewed the farmer whether he vaccinated continuously, intermittently, or not, against BHV-1 (before 1996, during 1996-1997 and in 1998), or whether he did not know the BHV-1-vaccination status. Last, the densities of the cattle and of the herds in the municipalities were determined by dividing the number of cattle and herds, respectively, by the effective agricultural land (Anonymous, 1997).

\subsection{Assessment of risk factors associated with BHV-1-seropositivity}

Analyses were performed using Stata ${ }^{\circledR}$, Version 8.0/SE (Stata Corporation, Texas, USA, 2003). Cluster-specific random-effects logistic-regression models were implemented. In these random-effects models, the intra-cluster (intra-herd) correlation is assumed to arise from natural heterogeneity (in the success probability) across clusters. Likelihood inference in this type of model proceeds by integrating over the distribution of random effects to derive the marginal likelihood, which can be done practically by numerical integration (Gaussian quadrature, for instance). Thus, the cluster effects are removed by assuming that they are realizations of a random variable.

The Stata ${ }^{\circledR}$ procedure gllamm was used for implementing these cluster-specific random-effects logistic-regression models. Gllamm ("generalized linear and latent mixed models") is a Stata command that fits a class of multilevel latent-variable models including multilevel generalized linear mixed models. Recently, adaptive quadrature has been proposed for multilevel models (Rabe-Hesketh et al., 2002). This allows using adaptive Gaussian quadrature to approximate the likelihood, which should be preferred to simple Gaussian quadrature in general (Lesaffre and Spiessens, 2001), as demonstrated also in the case of the BHV-1 survey (Speybroeck et al., 2003).

In a first descriptive analysis, a random-effects logistic-regression model investigated the risk factors for purchase-missingness (yes/no). 
The data were further analysed in two steps. First, bivariable analyses were conducted to compare the seroprevalence to each recorded risk factor. The significance of categorical variables was tested using Pearson's $\chi^{2}$-test for independence. Continuous variables were compared using a Wilcoxon rank-sum test. Second, risk factors for BHV-1-seroprevalence were assessed using logistic multiple-regression. All animal records with one or more missing covariate or response values were deleted (complete case analysis). Multicollinearity amongst putative risk factors was assessed using Spearman rank correlation coefficients, Pearson correlation coefficients, unpaired $t$-test, ANOVA, Kruskall-Wallis test and Wilcoxon rank-sum test. One variable in a pair was dropped if $R>|0.8|$ or if $P<0.05$.

The model-selection strategy consisted in considering all two-by-two interactions with a biologically meaningful interpretation and linear and second-order (quadratic) factors in a forward stepwise-selection procedure. The polynomial predictor variables were centred to avoid problems of multicollinearity (Neter et al., 1996). The inference criterion used to compare the models under consideration was their ability to predict the observed data. The Akaike information criterion (AIC) (Akaike, 1974) which penalizes the -2 log likelihood by adding twice the number of estimated parameters (Lindsey, 2004) was used. Smaller AIC values were thus to be preferred (Lindsey, 1997). The AIC obtained with a model including the intercept $b_{0}$ only was used as reference. The 'best' model was considered to be the model characterized by the smallest AIC.

\section{Results}

\subsection{Data exploration}

A general overview of the sample is provided in Table 1. At the animal level, there were missing values for the exposures age (26), sex (14) and purchase (2091), and for the BHV-1-test result (36). The median herd size was 19 overall, 60 for dairy herds, 59 for mixed herds and seven for beef herds. The average age (years) of the animals was lowest in beef herds (2.8), higher for mixed herds (2.9) and highest for dairy herds (3.1).

\subsection{Missing covariate observations}

The proportion of missing covariate observations for the variable purchase was considerable with an average of $27 \%$ per herd. The age of the animal and the BHV-1-test result were associated positively with missing values for purchase (Table 2). Exploration of the missingness (absence) of the variable purchase, by herd size, revealed that the largest herd (a beef herd with size 264) was most affected; 98\% (259/264) of its animal records lacked the purchase information (Fig. 1). Omission of this herd lead to different logistic regression results with age of animals and herd size being, respectively, positively and negatively associated with missing values for purchase (Table 2). The effect of BHV-1-test result became non-significant at the $5 \%$ level. The within-herd seroprevalence for this herd was $80 \%(212 / 264)$. 
Table 1

Variables and seroprevalence to bovine herpesvirus-1 in Belgian cattle, 1998

\begin{tabular}{|c|c|c|c|c|}
\hline \multirow[t]{2}{*}{ Risk factor } & \multirow[t]{2}{*}{ Category level } & \multirow[t]{2}{*}{ Number of observations } & \multicolumn{2}{|c|}{ Seroprevalence } \\
\hline & & & $\%$ & $95 \% \mathrm{CI}$ \\
\hline \multirow[t]{2}{*}{ Sex } & Cows & 9920 & 36 & 30,42 \\
\hline & Bulls & 1314 & 34 & 25,43 \\
\hline \multirow[t]{2}{*}{ Purchased } & No & 6815 & 33 & 26,39 \\
\hline & Yes & 2349 & 33 & 26,40 \\
\hline \multirow[t]{4}{*}{ Age (months) } & $\mathrm{Q} 1^{\mathrm{a}}(0.07,14.5)^{\mathrm{b}}$ & 2804 & 24 & 18,30 \\
\hline & Q2 (14.6, 29.5) & 2809 & 29 & 22,36 \\
\hline & Q3 $(29.6,48.1)$ & 2800 & 40 & 33,48 \\
\hline & Q4 (48.2, 192) & 2835 & 51 & 44,59 \\
\hline \multirow[t]{3}{*}{ Herd type } & Dairy & 4152 & 35 & 25,44 \\
\hline & Mixed & 3709 & 42 & 31,53 \\
\hline & Beef & 3387 & 31 & 21,41 \\
\hline \multirow[t]{4}{*}{ Herd size (number of animals) } & Q1 $(1,47)$ & 2814 & 26 & 21,31 \\
\hline & Q2 $(48,79)$ & 2867 & 33 & 24,41 \\
\hline & Q3 $(80,114)$ & 2830 & 45 & 32,57 \\
\hline & Q4 $(115,264)$ & 2737 & 41 & 23,59 \\
\hline \multirow[t]{4}{*}{ Density of cattle $\left(\left(\right.\right.$ cattle $\left.\left./ \mathrm{km}^{2}\right) / 100\right)$} & Q1 (34.01, 216.0) & 2752 & 32 & 21,42 \\
\hline & Q2 (216.0, 262.6) & 2840 & 36 & 25,47 \\
\hline & Q3 $(262.7,333.4)$ & 2760 & 34 & 23,45 \\
\hline & Q4 (333.5, 997.6) & 2896 & 42 & 29,56 \\
\hline \multirow[t]{4}{*}{ Density of herds $\left(\left(\right.\right.$ herds $\left.\left./ \mathrm{km}^{2}\right) / 100\right)$} & Q1 (0.83, 3.05) & 2792 & 33 & 21,46 \\
\hline & Q2 $(3.06,3.91)$ & 2831 & 40 & 29,51 \\
\hline & Q3 $(3.92,4.79)$ & 2867 & 33 & 21,45 \\
\hline & Q4 (7.80, 10.06) & 2758 & 38 & 26,51 \\
\hline
\end{tabular}

${ }^{\mathrm{a}} \mathrm{Q}$, quartile.

b Minimum, maximum category value.

\subsection{Seroprevalence risk factor analysis}

\subsubsection{Bivariable analysis of the recorded risk factors}

The distribution of risk factors between seronegative and seropositive cattle is summarized in Table 1. It shows that there were no obvious differences between the seroprevalence of cows and bulls and between homebred or purchased animals. The data

Table 2

Random-effects logistic-regression model of missing data for the animal-level variable "purchase (yes/no)" in 11,284 Belgian cattle from 309 herds, 1998

\begin{tabular}{|c|c|c|c|c|c|c|c|c|}
\hline \multirow[t]{2}{*}{ Risk factor } & \multicolumn{4}{|c|}{ Model with outlying herd } & \multicolumn{4}{|c|}{ Model without outlying herd } \\
\hline & OR & S.E. & $P$-value & $95 \% \mathrm{CI}$ & OR & S.E. & $P$-value & $95 \% \mathrm{CI}$ \\
\hline BHV-1-test result $(0=$ negative $)$ & 1.77 & 0.231 & $<0.001$ & $1.37,2.28$ & 1.65 & 0.46 & 0.075 & $0.95,2.88$ \\
\hline $\begin{array}{l}\text { Herd size (number of cattle } \\
\text { on the premises) }\end{array}$ & 1.00 & 0.006 & 0.88 & $0.99,1.01$ & 0.99 & 0.004 & 0.021 & $0.98,1.00$ \\
\hline Age (months) & 1.02 & 0.001 & $<0.001$ & $1.01,1.02$ & 1.02 & 0.006 & 0.011 & $1.00,1.03$ \\
\hline
\end{tabular}




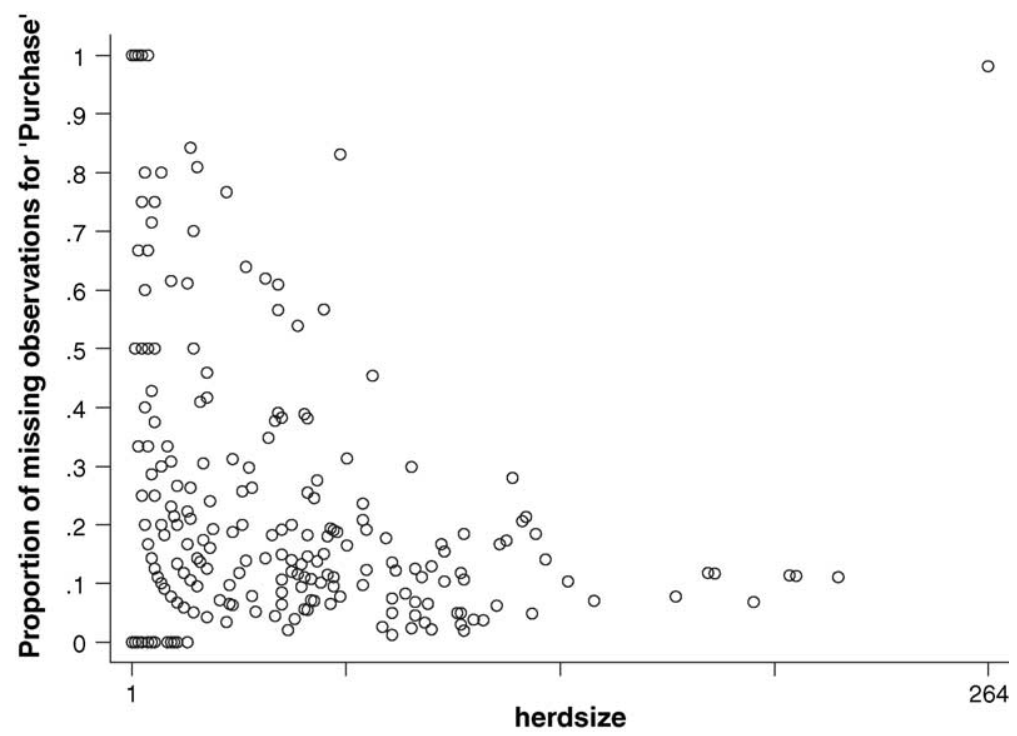

Fig. 1. Scatterplot of the herd-specific proportion of missing observations for 'purchase', by herd size.

suggested that seroprevalence might be related to age and herdsize in a quadratic way, prompting the inclusion of quadratic terms.

\subsubsection{Logistic-regression models}

No collinearity was discerned between the continuous covariates. The variable purchase was associated with sex $\left(\chi^{2}=41.05\right.$, d.f. $\left.=1, P<0.001\right)$; one in four cows was purchased compared to one in three bulls. But, purchase also was associated with herd type $\left(\chi^{2}=418.39\right.$, d.f. $\left.=2, P<0.001\right)$; in dairy herds, $17.2 \%$ of the cattle were purchased, compared to 23.6 and $40.3 \%$ for mixed and beef herds, respectively. The final model included the animal-level factors age, sex and origin (purchased or homebred), and the herd-level covariate herd size. An increasing (centred) age was a risk factor for seropositivity, but this effect levelled off at older age (significant negative quadratic term). Bulls were more at risk, compared to cows. A significant negative interaction was observed between purchase status and (centred) herd size and a positive one between the same variable and the square of (centred) herd size. The final model is shown in Table 3. The log likelihood and the AIC for this model estimating nine parameters were -3289.08 and 3307.08, respectively.

The final model defined the following equation:

$$
\begin{aligned}
\operatorname{logit}(\pi)= & -2.35+b_{i}+0.51 * \text { purchase }+0.32 * \text { sex }+0.040 * \text { herd size } \\
& -0.00020 * \text { herd size }{ }^{2}+0.042 * \text { age }-0.000090 * \text { age }^{2}-0.013 \\
& * \text { herd size } * \text { purchase }+0.000063 * \text { herd size }{ }^{2} * \text { purchase },
\end{aligned}
$$

in which $b_{i}$ is the herd-level, random intercept. 
Table 3

Final random-effects logistic-regression model in 11,284 Belgian cattle from 309 herds, 1998

\begin{tabular}{|c|c|c|c|c|}
\hline Risk factor & OR & S.E. & $P$-value & $95 \% \mathrm{CI}$ \\
\hline Age (months) & 1.04 & 0.002 & $<0.001$ & $1.04,1.05$ \\
\hline $\operatorname{Age}^{2}$ (months) & 0.99991 & 0.00003 & 0.005 & $0.9998,0.9999$ \\
\hline $\operatorname{Sex}(\operatorname{cow}=0)$ & 1.37 & 0.165 & 0.009 & $1.08,1.74$ \\
\hline Purchase $($ no $=0)$ & 1.67 & 0.203 & $<0.001$ & $1.32,2.12$ \\
\hline Centred herd size & 1.04 & 0.006 & $<0.001$ & $1.03,1.05$ \\
\hline Centred herd size ${ }^{2}$ & 0.9997 & 0.00005 & $<0.001$ & $0.9996,0.9999$ \\
\hline Interaction centred herd size by purchase & 0.987 & 0.003 & 0.001 & $0.979,0.994$ \\
\hline Interaction centred herd $\operatorname{size}^{2}$ by purchase & 1.00 & 0.00002 & 0.014 & $1.00,1.00$ \\
\hline
\end{tabular}

Herd size, number of animals on the premises.

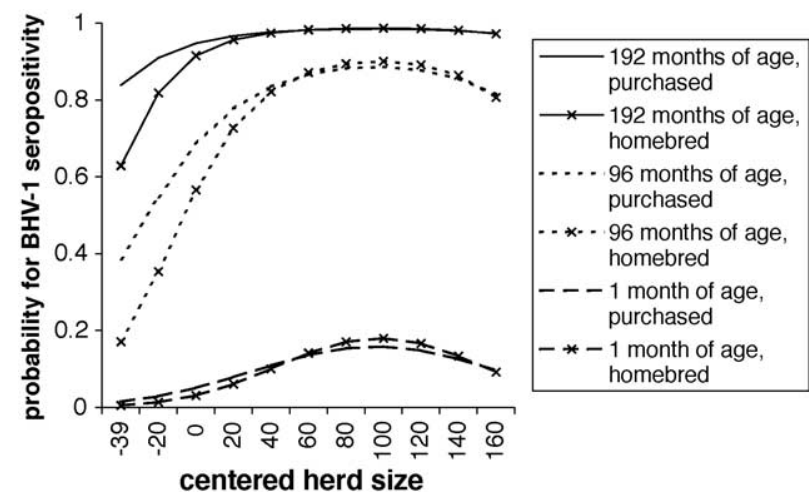

Fig. 2. Relation between centred herd size and the probability for bulls to be seropositive to BHV-1, for different ages and for purchase (yes/no), by herd size.

Figs. 2 and 3 plot this equation, respectively, for bulls and cows, by herd size. A grid of observed herd sizes was chosen. These figures first show that age is a risk factor for BHV-1seropositivity. Second, bulls have a persistently higher probability to be BHV-1seropositive, compared to cows. Third, these figures show that the effects of the risk factors

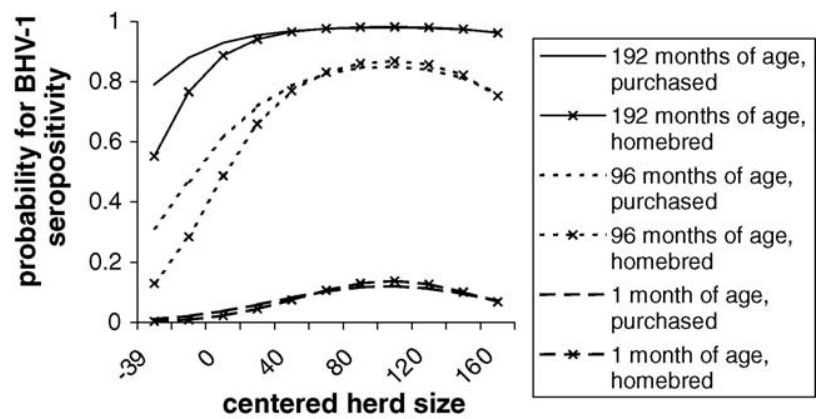

Fig. 3. Relation between centred herd size and the probability for cows to be seropositive to BHV-1, for different ages and for purchase (yes/no), by herd size. 
purchase and herd size interact. The significant negative interaction between purchase status and herd size plus the positive interaction between the same variable and the square of herd size means that its overall effect was different for smaller and larger herds. For small herds ( $\leq 50$ cattle on the premises), purchased cattle were more at risk, compared to homebred ones (curve of purchased origin persistently higher than those of homebred origin), and larger herd size was also a risk factor (positive slope). For larger herds, these overall effects were not observed.

\section{Discussion}

\subsection{Missing values for purchase}

Most of the missing purchase data were for a single beef herd; this herd was the largest in the database. As demonstrated graphically, there were hardly any other herds of this size in the sample. Consequently, relevant information is lacking to conclude anything with confidence regarding such large beef herds. More importantly, omission of this outlying herd led to a subsample for which purchase-missing values did not depend any more on the BHV-1 test result. The purchase-missing values were caused by a technical problem while conducting the survey; for animal-level identification, the animals' working eartag numbers were noted-not their official ones. The advantage of the former ones is higher readability. Unfortunately, these working eartag numbers were not indexed in SANITEL. We hypothesised that the complete case analysis, as implemented, can be considered to be unbiased with respect to purchase being a BHV-1-seroprevalence risk factor or not (Zhao et al., 1996). The missing values thus only constitute a sample-size reduction.

\subsection{Risk factors for BHV-1-seropositivity}

To our knowledge, no other cluster-sample surveys are reported in the literature investigating risk factors for BHV-1-seropositivity at the animal-level, incorporating an unvaccinated cattle population and herds of all types. Speybroeck et al. (2003) analysed a subset of the present BHV-1-survey data to exemplify population-averaged and clusterspecific models.

The most-important finding in this study was the interaction between the effects of the risk factors purchase and herd size. For herds up to 50 animals, purchased status and larger herd size were risk factors, whereas these effects were not observed for larger herds. The interpretation of this marked difference in odds for cattle in smaller herds considers the biology of BHV-1 infection. A key epidemiological feature of BHV-1 is its contagion-a group effect of the dependent variable. It is a fundamental dynamic of communicable disease (Susser, 1994b; De Jong, 1995), and explains why infections mirror the clustering of animal populations. This is also the case in the BHV-1 survey, because the intra-cluster correlation was 0.64 (Speybroeck et al., 2003), which is high. Once the infection is introduced in a herd, it quickly spreads within-herd, to purchased and to homebred herd mates. The probability of an animal being sampled in the serological silent period-about 7-10 days (Davies and Carmichael, 1973; Kramps et al., 1994)—is low. Also, once 
infected, most cattle have lifelong antibodies (Chow, 1972; Kahrs, 1977). Consequently, seroprevalence figures — and consequently the statistical modelling outcome-are a postepidemic snapshot and non-informative regarding the within-herd index case and the within-herd infection dynamic. In larger herds, the contagion of homebred herd mates could have masked the importance of purchased index cases as direct sources of virus introduction. From a biological perspective, purchase status would appear to be a direct risk factor for herds of any size. That purchase was a major direct risk factor is consistent with other studies (Wentink et al., 1993; van Schaik et al., 1998).

Also, the herd size was a risk factor for BHV-1-seropositivity. In smaller herds, the number of susceptible animals is smaller throughout the year, so infection might not be maintained. The risk of BHV-1 transmission among cattle within infected herds is also higher for larger herds, because they usually have loose-housing barns (creating more contact between infected and susceptible animals). These herds possibly also have more visits by animal handlers (farmers, inseminators, veterinarians, traders) (van Wuijckhuise et al., 1998).

Bulls were more at risk of being seropositive compared to cows; this finding is new. It could be that bulls have more 'risky' contacts compared to cows. An example of such contacts is more-frequent participation of bulls in cattle shows. Also, bulls possibly display a more-risky behaviour than cows. Cattle that escape and mingle with other cattle are a risk for virus introduction into herds (van Schaik et al., 2001). If bulls escape and mingle more often than cows, this would explain our findings.

Last, increasing age was a risk factor for seropositivity, but this effect levelled off at higher age. This result is consistent with other studies (van Wuijckhuise et al., 1993; Kadohira et al., 1996) and was expected because age is a surrogate measure of amount of exposure time. This always happens with life-long seropositivity, as is the case for BHV-1 infection (Chow, 1972; Kahrs, 1977).

In conclusion, prevention of BHV-1-seropositivity should primarily focus on purchase that directly introduces virus into the herds. The farmer should prevent direct and lengthy animal contacts in order to considerably reduce the risk of BHV-1 introduction. Only certified BHV-1 uninfected cattle should be purchased. After purchase of cattle, quarantine measures should be rigorously applied. Uninfected cattle should further be prevented from coming into contact - directly and indirectly - with cattle originating from larger herds that are not certified BHV-1 free, or bulls or older cattle with unknown BHV-1 infection status. Due to sample limitations, these conclusions cannot be extrapolated to large beef herds.

\section{Acknowledgements}

The BHV-1 survey was supported by the Fund for Animal Health and Production, Federal Public Service Health, Food Chain Safety and Environment, Belgium.

\section{References}

Akaike, H., 1974. A new look at the statistical model identification. IEEE Trans. Autom. Control AC-19, 716-723. Anonymous, 1997. Belgian Agricultural Statistics Yearbook. 
Boelaert, F., Biront, P., Soumare, B., Dispas, M., Vanopdenbosch, E., Vermeersch, J.P., Raskin, A., Dufey, J., Berkvens, D., Kerkhofs, P., 2000. Prevalence of bovine herpesvirus-1 in the Belgian cattle population. Prev. Vet. Med. 45, 285-295.

Cameron, A.R., Baldock, F.C., 1998. Two-stage sampling in surveys to substantiate freedom from disease. Prev. Vet. Med. 34, 19-30.

Chow, T.L., 1972. Duration of immunity in heifers inoculated with infectious bovine rhinotracheitis virus. J. Am. Vet. Med. Assoc. 160, 51-54.

Davies, D.H., Carmichael, L.E., 1973. Role of cell-mediated immunity in the recovery of cattle from primary and recurrent infections with infectious bovine rhinotracheitis virus. Infect. Immun. 8, 510-518.

De Jong, M.C., 1995. Mathematical modelling in veterinary epidemiology: why model building is important. Prev. Vet. Med. 25, 183-193.

de Wit, J.J., Hage, J.J., Brinkhof, J., Westenbrink, F., 1998. A comparative study of serological tests for use in the bovine herpesvirus 1 eradication programme in The Netherlands. Vet. Microbiol. 61, 153-163.

Donald, A., Donner, A., 1987. Adjustments to the Mantel-Haenszel $\chi^{2}$ statistic and odds ratio variance estimator when the data are clustered. Stat. Med. 6, 491-499.

Elbers, A.R., Schukken, Y.H., 1995. Critical features of veterinary field trials. Vet. Rec. 136, 187-192.

Haine, D., Boelaert, F., Pfeiffer, D., Saegerman, C., Lonneux, J.F., Losson, B., Mintiens, K., 2004. Seroprevalance and risk-mapping of bovine hyperdermosis in Belgian cattle herds. Prev. Vet. Med. 65, 93-104.

Kadohira, M., McDermott, J.J., Shoukri, M.M., Thorburn, M.A., 1996. Assessing infections at multiple levels of aggregation. Prev. Vet. Med. 29, 161-177.

Kahrs, R.F., 1977. Infectious bovine rhinotracheitis: a review and update. J. Am. Vet. Med. Assoc. 171, 10551064.

Koopman, J.S., Longini, I.M.J., Jacquez, J.A., Simon, C.P., Ostrow, D.G., Martin, W.R., Woodcock, D.M., 1991. Assessing risk factors for transmission of infection. Am. J. Epidemiol. 133, 1199-1209.

Kramps, J.A., Magdalena, J., Quak, J., Weerdmeester, K., Kaashoek, M.J., Maris-Veldhuis, M.A., Rijsewijk, F.A., Keil, G., van Oirschot, J.T., 1994. A simple, specific, and highly sensitive blocking enzyme-linked immunosorbent assay for detection of antibodies to bovine herpesvirus 1. J. Clin. Microbiol. 32, 21752181.

Lesaffre, E., Spiessens, B., 2001. On the effect of the number of quadrature points in a logistic random-effects model: an example. Appl. Statist. 50, 325-335.

Lindsey, J.K., 1997. Applying Generalised Linear Models. Springer-Verlag, New York.

Lindsey, J.K., 2004. Introduction to Applied Statistics: A Modelling Approach. Oxford University Press, Oxford.

Neter, J., Kutner, M.H., Nachtsheim, C.J., Wasserman, W., 1996. Applied Linear Statistical Models. McGrawHill/Irwin, Chicago.

O.I.E., 2004. International Terrestrial Animal Health Code. Office International des Epizooties, Paris, http:// www.oie.int/.

Rabe-Hesketh, S., Skrondal, A., Pickles, A., 2002. Reliable estimation of generalized linear mixed models using adaptive quadrature. Stata J. 22, 1-21.

Rothman, K.J., 1990. A sobering start for the cluster busters' conference. Am. J. Epidemiol. 132, S6-S13.

Schukken, Y.H., Casella, G., van den Broek, J., 1991. Overdispersion in clinical mastitis data from dairy herds: a negative binomial approach. Prev. Vet. Med. 10, 239-245.

Speybroeck, N., Boelaert, F., Renard, D., Burzykowski, T., Mintiens, K., Molenberghs, G., Berkvens, D., 2003. Design-based analysis of surveys: a bovine herpesvirus 1 case study. Epidemiol. Infect. 13, 991-1002.

Susser, M., 1994a. The logic in ecological. Part I: the logic of analysis. Am. J. Public Health 84, 825-829.

Susser, M., 1994b. The logic in ecological. Part II: the logic of design. Am. J. Public Health 84, 830-835.

van Schaik, G., Dijkhuizen, A.A., Huirne, R.B., Schukken, Y.H., Nielen, M., Hage, H.J., 1998. Risk factors for existence of bovine herpes virus 1 antibodies on nonvaccinating Dutch dairy farms. Prev. Vet. Med. 34, 125-136.

van Schaik, G., Schukken, Y.H., Nielen, M., Dijkhuizen, A.A., Benedictus, G., 2001. Risk factors for introduction of BHV1 into BHV1-free Dutch dairy farms: a case-control study. Vet. Q. 23, 71-76.

van Wuijckhuise, L., Bosch, J., Franken, P., Frankena, K., Elbers, A.R., 1998. Epidemiological characteristics of bovine herpesvirus 1 infections determined by bulk milk testing of all Dutch dairy herds. Vet. Rec. 142, 181-184. 
van Wuijckhuise, L., Bosch, J., Franken, P., Hage, J., Verhoeff, J., Zimmer, G., 1993. The prevalence of infectious bovine rhinotracheitis (IBR) in the Netherlands. In: Elbers, A.R.W. (Ed.), Proceedings of the Dutch Society for Veterinary Epidemiology and Economics (VEEC). pp. 7-15.

Wentink, G.H., van Oirschot, J.T., Verhoef, J., 1993. Risk of infection with bovine herpesvirus 1 (BHV1): a review. Vet. Q. 30-33.

Zhao, L.P., Lipsitz, S., Lew, D., 1996. Regression analysis with missing covariate data using estimating equations. Biometrics 52, 1165-1182. 